\title{
WIEDERGABE VON LITERARISCHEN VORNAMEN IN DEUTSCH-POLNISCHER ÜBERSETZUNG (AM BEISPIEL DES ROMANS BUDDENBROOKS VON THOMAS MANN)
}

\author{
ELIZA PIECIUL \\ Adam-Mickiewicz-Universität-Poznań
}

\begin{abstract}
The article discusses the question of the position of proper names (first names) in literary German-Polish translation. The empirical basis consists of first names from the novel by Thomas Mann (The Buddenbrooks) and its Polish translation. The first part of the article contains a theoretical introduction in the field of onomastics and discusses the nature and specific features of first names (1). In the next step different strategies of rendering of literary proper names are presented (2). The empirical analysis (3) shows the two main translation strategies: transcribing and adapting of literary first names, and their consequences for the target language reception. The article sums up the analysis of chosen first names presenting linguistic and cultural conditions for rendering of proper names in literary translation (4).
\end{abstract}

Der vorliegende Aufsatz möchte die Ergebnisse der Arbeit: „Literarische Personennamen in deutsch-polnischer Translation. Eine kontrastive Studie aufgrund der Prosawerke von Thomas Mann (Buddenbrooks, Der Zauberberg, Doktor Faustus)" zusammenfassen, die im Dezember 2000 von der Neuphilologischen Fakultät der Adam-Mickiewicz-Universität in Poznan als Dissertation angenommen wurde. Einer kurzen Darstellung der VN-Spezifik (1) und der translatorischen Tendenzen in der Wiedergabe von literarischen Vornamen (2) folgt eine Analyse dieses Problems in der polnischen Übersetzung der Buddenbrooks $^{1}(3)$ und eine kurze Zusammenfassung der Analyse der Wiedergabe von literarischen Vornamen (4).

\footnotetext{
${ }^{1}$ Der Roman eignet sich besonders gut zur Beschreibung der Spezifik der deutsch-polnischen Vornamenwiedergabe, denn es gibt dort zahlreiche Dialogpassagen (daher Anredeformen) und Hypokoristika, deren Wiedergabe für das zielseitige Nachvollziehen der ausgangsseitig beabsichtigten Gefüle und Reaktionen nicht ohne Bedeutung ist. Neben dem Namenmaterial aus den Buddenbrooks (B) werden im vorliegenden Aufsatz auch Belege aus den zwei anderen Romanen Thomas Manns ins Feld geführt (Der Zauberberg - Z, Doktor Faustus - F).
} 


\section{Zur Natur von Vornamen}

Der Vorname als individueller Namenteil unterscheidet die betreffende Person von anderen Mitgliedern der Familie und wird in einem Namengebungsakt von den Eltern verliehen. Da die Vornamengebung im Gegensatz zu Familiennamen, die den Namensträger als Mitglied einer bestimmten Familie kennzeichnen, nicht automatisch erfolgt, sind dabei bestimmte Selektionsprinzipien (Motive, Affekte, Zielvorstellungen) der Namenwahl von Bedeutung. Natürlich sind meistens mehrere Motive in einem Einzelfall wichtig, wobei man damit rechnen muß, daß die danach Befragten ${ }^{2}$ oft nicht in der Lage sind, das dominierende Motiv anzugeben (vgl. Seibicke 1982:116).

Da die Namengebung in einem formellen Namengebungsakt erfolgt, der an bestimmte Lebenssituationen gebunden ist (z.B. Taufe), schriftlich fixiert wird und zum Gebrauch eines bestimmten Namens verpflichtet, so kann die Namenwahl nicht willkürlich geschehen. Auch hier zeichnet sich ein Unterschied zu Familiennamen deutlich ab, da der Vornamenkanon eine ,relativ geschlossene Liste" ist (Zimmer 1981:67), während Familiennamen eine offene Liste bilden. Die Gebundenheit der Vornamen an das Zivilrecht des jeweiligen Staates verursacht $^{3}$, daß die Vornamen "sichere Informationen über die nationale Herkunft des Trägers" liefern (ebd., 103), was in der literarischen Namengebung als ein Zeichen der nationalen und lokalen Zuordnung gebraucht und verstanden wird.

\subsection{Formen der Vornamen}

Die besondere Stellung der Vornamen in der alltäglichen Kommunikation verursacht, daß sie in unterschiedlichen Formen auftreten: Voll-, Kurz- und Koseformen. Als Vollformen werden im vorliegenden Aufsatz VN-Formen verstanden, die nicht durch Kürzung als einer längeren Ursprungsform entstanden und nicht durch Suffixe erweitert sind (vgl. Seibicke 1982:96). Zu Kurzformen zählen alle Arten der Kürzung von Vollformen, auch solche mit zusätzlichen Wortbildungsmitteln: Kurt zu Konrad, Klaus zu Nikolaus. Sie entstehen in der Umgangssprache, können aber durch häufigen Gebrauch zu vollwertigen Namen

\footnotetext{
${ }^{2}$ Namenwahlmotive werden als eine soziologische Frage untersucht, z.B.: In welchen gesellschaftlichen Kreisen überwiegen bestimmte Motive? Wie sind die Unterschiede zu erklären? Siehe die Untersuchung von Shin, Schichtenspezifische Faktoren der Vornamengebung. Empirische Untersuchung der 1961 und 1976 in Heidelberg vergebenen Vornamen (1980). Daß die Motivation der Vornamengebung auch etwas von der Kulturgeschichte aussagen kann, zeigt eine interessante Arbeit von Wolffsohn/Brechenmacher: Die Deutschen und ihre Vornamen. 200 Jahre Politik und offentliche Meinung (1999).

${ }^{3}$ Zum Namenrecht siehe vor allem die Studie von Seutter (1996); vgl. auch Bubak (1998).
} 
werden, deren Herkunft aus einer Vollform nicht mehr empfunden wird, z.B.: Heinz von Heinrich (vgl. Witkowski 1964:43). Kosenamen (Hypokoristika) sind expressive Namenformen mit kosender Bedeutung, die mit Hilfe sog. Verkleinerungs- oder Deminutivsuffixe gebildet werden, z.B. Rudi, Karlchen, Staś, Tommy usw. Oft liegen den Kosenamen Kurzformen (Käthchen zu Käthe) aber auch Vollformen (Güntherchen zu Günther) zugrunde (vgl. Witkowski 1964:42).

Die Grenzen zwischen den Formen sind oft verschwommen: Zahlreiche Kurznamen können als Koseformen gebraucht werden, während Kosenamen Kurznamen sein können, aber nicht müssen (vgl. auch Jakus-Borkowa 1986:35). Eine klare Trennung zwischen Kurz- und Koseformen ist also nicht möglich, weil einerseits die Bildung von Koseformen sehr oft mit der Kürzung des zugrunde liegenden Namens einhergeht, andererseits Kurzformen auch ohne Deminutivsuffixe als Koseformen gebraucht und verstanden werden. (Seibicke 1982:97).

Die Einteilung in Voll-, Kurz- und Koseformen basiert auf der extralinguistischen Funktion der jeweiligen Vornamenform (vgl. Grodziński 1973:117). Taufformen kommen nämlich vorwiegend in Dokumenten, in offiziellen Beziehungen und Situationen vor und werden relativ selten in der Alltagskommunikation gebraucht. In der Umgangsprache (als Anredeform unter Familienmitgliedern, Verwandten und Freunden) verwendet man dafür Kurz- und Koseformen (vgl. Umińska-Tytoń 1985:115).

\section{Literarische Vornamen in deutsch-polnischer Übersetzung}

Die sprachuniversale Tatsache, daß Vornamen sowohl im täglichen Sprachgebrauch als auch in literarischen Werken neben den für Vornamen und Familiennamen gemeinsamen auch spezifische stilistische Funktionen haben, ist für die Abgrenzung des Problembereiches nicht unwichtig (z.B. Hypokoristika als Ausdruck der emotionalen Nähe). Vornamen sind auch Bestandteil der Ausgangskultur und in der Übersetzung können sie somit zu Zeichen werden, die die AS-Welt im übersetzten Text aktualisieren, oder sie können infolge der Adaptation eine Integration der AS-Welt mit der zielspracheigenen Ausdruckseite bewirken (vgl. Kalverkämper 1995:1021).

\subsection{Transkription und Adaptation/Substitution der Vornamen}

Die Entscheidung, einen AS-Vornamen oder seine ZS-Variante im ZS-Text zu gebrauchen, hat nicht nur eine ,punktuelle", mikrostrukturelle Bedeutung für den Translat, vielmehr ist der ,ganze Text, der Rezeptionsprozeß selbst (...) 
betroffen" (Kalverkämper 1995:1021). Der Übersetzer eines literarischen Textes steht vor der Alternative:

George mit George oder Georg zu übersetzen. In beiden Fällen liegen Übersetzungen vor. (Neubert 1973:75)

Der Begriff 'Übersetzung' - wie das obige Zitat zeigt - erscheint oft auch in bezug auf die Wiedergabe von Vornamen. Im vorliegenden Aufsatz wird die VN-Wiedergabe in Transkription, Adaptation und Substitution unterschieden. Im besprochenen Namenkorpus (literarische Vornamen in den Buddenbrooks) dominieren zwei Tendenzen der Wiedergabe: Transkription der Vornamen als Bewahrung ihrer Originalform oder Adaptation als Anwendung einer zielseitigen Namenvariante ${ }^{4}$. Man findet wenige Belege für Substitution, die in bezug auf Vornamen als Wiedergabe des AS-Vornamens mit einem anderen Vornamen verstanden 5 .

Die Frage, welche Formen der Vornamen man zielseitig anwenden soll, wird von verschiedenen Standpunkten her beantwortet. Güttinger, Vertreter einer traditionellen und radikalen Option, plädiert für eine konsequente Einverleibung fremder Vornamen. Fremde Vornamen, die es auf deutsch auch gibt, solle man in ihrer deutschen Form wiedergeben, meint Güttinger, und bekräftigt es mit den Worten: „Gute Übersetzer haben es jedenfalls noch immer so gehalten" (1963:85). Er hält Vornamen für ein Sprachelement, das neben Appellativen übersetzt werden soll, wodurch auch „Miss Atmosphäre” programmatisch ignoriert wird (ebd., 85), also die Tatsache, daß einverleibte Vornamen das Lokalkolorit des Ausgangstextes beeinträchtigen.

Man kann Güttingers Appell: „Vorrang der Muttersprache” (ebd., 218) als Zeichen des traditionellen Übersetzungsansatzes verstehen, der den Zeiten entstammt, in denen Leser von Übersetzungen aus dem Englischen „mit dem Schluss - $e$ [beim VN George] sicher nichts anzufangen" wußten (ebd.). So sahen sich Übersetzer verpflichtet, das Fremde durch das Bekannte zu ersetzen ${ }^{6}$. Die Adaptation der Vornamen bedeutet aber einen Verzicht auf ihre zuordnende Funktion.

Heutzutage hat sich das Wissen, über das Leser von Übersetzungen verfügen, infolge der Globalisierung wesentlich verändert. „Und eben das ist eine der Voraussetzungen für das kreative Mitwirken des Lesers am Übersetzen" (Mark-

${ }^{4}$ Adaptation umfaßt gleichzeitig eine phonetische und/oder graphemische Anpassung an die Zielsprache (Moryc fur [Max und] Moritz, in Z) wie auch die bereits etablierten einzelsprachlichen Äquivalente (Maurycy fur Moritz, in B), die als Namendubletten bezeichnet werden (vgl. Krüger 1997:474). Der Gebrauch der parallelen Namenvarianten wird als Adaptation und nicht Substitution verstanden (anders als bei Debus 1997:399).

${ }^{5}$ Dies betrifft vorwiegend mundartliche Vornamenformen, die mit den standardsprachlichen ZS-Formen substituiert werden (Benedykt anstatt Bendix, s. 3.1).

${ }^{6}$ Also den Autor an den Leser bringen (vgl. Schleiermachers Antithese, 1973:47). 
stein 1995:99). Das Fremde wirkt nicht mehr befremdend und man kann annehmen, daß gegenwärtige Leser zu größerem Risiko bereit sind und daß sie die fremde Welt in ihrer spezifischen Gestalt über das Medium der Übersetzung kennenlernen wollen. Dies bedeutet, daß man bei der Lektüre einer Übersetzung eher Überraschung als Bestätigung erwartet, so daß der moderne Übersetzer auf Verständnis der Leser rechnen kann, wenn sie mit der Fremdheit konfrontiert werden (vgl. Krusche 1985:139).

Die Entscheidung, Vornamen zu transkribieren, sorgt für die von Güttinger geschmähte „Miss Atmosphäre”, da bei der Wahl der Form George (wir bleiben beim Beispiel George/Georg) das englische Vornamensystem als Fremdheitseffekt erhalten bleibt (vgl. Neubert 1973:75). Der Vorname wird in diesem Ansatz nicht als ein zu übersetzendes Sprachelement, sondern als Bestandteil der Ausgangsprache und infolgedessen der Ausgangskultur, betrachtet, der deren Wirkung im übersetzten Text garantiert ${ }^{7}$. Die Fremdheitsatmosphäre wird hier als ein inhaltlich relevanter Bestandteil des übersetzten Textes aufgefaßt, die dank transkribierter Vornamen auch in der Übersetzung bewahrt werden kann. Man kann Vornamen als sprachlich fixierte, historische und kulturelle Zeichen der Fremdheit auffassen, die nicht unbedingt Ergebnis der Unübersetzbarkeit dieser Elemente sind (vgl. Legeźyńska 1983:80). Jene „Fremdheitsatmosphäre” soll man dabei nicht als eine vage, undefinierbare Stimmung verstehen, die man auch in der Übersetzung kreieren soll. Gemeint ist eher „die in der Sprache aufbewahrte Geschichte und Kultur der jeweiligen Sprachgemeinschaft" (Markstein 1995:98). Transkribierte Vornamen sind Zeichen der fremden Welt in der Zielsprache und erfüllen dabei ohne Hindernisse die Funktion der nationalen Zuordnung. Die Adaptation der AS-Vornamen führt dagegen zur Schwächung ihres Ausdrucks oder zur Annahme, daß es sich beim Namensträger eines eingebürgerten Vornamens um den Vertreter der ZS-Welt handelt (vgl. Newmark 1981:70).

Da jedoch in der polnischen Sprache Eigennamen flektiert werden, kann Transkription im Falle fremder, komplizierter Namensformen zu beträchtlichen Schwierigkeiten führen (vgl. Kurecka 1970:88). Es reicht also nicht aus, die Methode der Transkription als "die" Methode der Vornamenwiedergabe zu preisen. Auch die strukturelle Seite der Übersetzung - hier spezifisch der deutsch-polnischen - muß berücksichtigt werden. Aus diesem Grund (und nicht ausschließlich um des „Vorrangs der Muttersprache” willen) entscheiden sich Übersetzer für zielseitige Namenvarianten (auch um den Preis des Lokalkolori-

\footnotetext{
${ }^{7}$ Nowakowska-Kempna spricht sogar von der Pflicht der Transkription, weil Vornamen als integraler Teil der AS-Welt nur auf diesem Weg dieser Welt in der Rezeption des ZS-Lesers weiterhin angehören können (vgl. 1978:112).
} 
tes), die in der Zielsprache reibungslos funktionieren. Die bewußte Wahl der Transkription, die ausgangsseitige Konnotationen bewahren soll, wird somit durch die Forderungen des Sprachsystems relativiert, und der Übersetzer bewegt sich oft in einem begrenzten Raum zwischen seiner freien Entscheidung und dem Zwang der Struktur.

\subsection{Wiedergabe von Hypokoristika}

In der für Vornamen typischen Unterscheidung zwischen den Voll-, Kurz- und Koseformen findet die emotionale Wertung statt. Der Gebrauch einer Vollform vs. einer Kurzform kann z.B. den Vertrautheitsgrad oder gewisse Emotionen zum Ausdruck bringen, was sich natürlich primär im realen Sprachgebrauch manifestiert. Diese Eigenschaft wird natürlich auch in der literarischen Namengebung ausgenutzt: Koseformen bringen das emotionale Verhältnis zu den kreierten Figuren zum Ausdruck (beispielsweise um die Nähe zum Protagonisten hervorzuheben); sie betonen auch gegenseitige Beziehungen zwischen den Figuren (vgl. Niesporek 1986:119) ${ }^{8}$.

Der konnotative Wert eines Vornamens (also jeweiliger Vornamenform: Voll-, Kurz- oder Koseform) und dessen Wiedergabe sind für eine adäquate Übersetzung nicht unwichtig9. Der Übersetzer muß vor allem entscheiden, ob und mit welchen ZS-Mitteln er die Ausdrucksfunktion der Hypokoristika bewahren kann. Es besteht natürlich die Gefahr, daß gewisse Kose- oder Kurzformen in der transkribierten Übersetzung nicht in ihrer Funktion erfaßt werden können (Gebrauch der Koseform setzt bestimmte Reaktionen beim Leser voraus), weil sie z.B. als solche nicht empfunden werden (vgl. Cieślikowa 1996:314).

Der Übersetzer sitzt oft in einer Zwickmühle: Entweder bewahrt er die Koseform mit Hilfe von zielseitigen Sprachmitteln, oder er verzichtet auf die Wiedergabe der Namenstilistik und transkribiert den Vornamen. In seltenen Fällen sind AS-Hypokoristika auch in der Zielsprache als solche erkennbar (beispielsweise Adri, Rudi in Doktor Faustus).

${ }^{8}$ Dies kann als Namenstilistik bezeichnet werden (vgl. Thies 1978:76).

${ }^{9}$ Es sind vor allem fiektierende Sprachen, die über eine enorme und oft irreführende Vielfalt der Diminutive verfügen. Beim deutsch-polnischen Vergleich macht sich die Problematik der Wiedergabe von Koseformen vor allem in Übersetzungen aus der polnischen Sprache bemerkbar. Aber auch die Übersetzung von deutschen Hypokoristika ist nicht weniger kompliziert, denn der Gebrauch zielseitiger Sprachmittel zur Bildung der Diminutiva kann den Rezeptionsprozeß beeinflussen. 


\section{Hans oder Janek? Zur Frage der Wiedergabe literarischer Vornamen}

\subsection{Einverleibung der deutschen Vornamen am Beispiel der Buddenbrooks}

Die allgemeine Tendenz der VN-Wiedergabe in den Buddenbrooks ist das adaptierende Verfahren. Es betont den „Vorrang der Muttersprache” (s.o.), so daß die Mehrheit der AS-Vornamen mit zielseitigen Formen ersetzt wird, auch wenn der Vorname über keine Namendublette in der Zielsprache verfügt. Auf diese Weise haben wir in den Buddenbrooks nicht nur mit der Adaptation zu tun, sondern auch mit der Substitution, vorwiegend von mundartlichen VNFormen (Corl wird zu Karol; Krischan zu Chrystian; Bendix zu Benedykt).

Der Roman Buddenbrooks kann als Beispiel eines translatorischen Verfahrens angesehen werden, in dem man Vornamen wie andere Sprachzeichen , übersetzt" und sie nicht als Elemente der AS-Welt betrachtet, die diese Welt in der Übersetzung vertreten sollen. Das adaptierende Verfahren führt dazu, daß die erzählte Geschichte den Eindruck erweckt (oder erwecken sollte), sie spiele sich „bei uns”, in der bekannten Umgebung ab. Figuren, die ursprünglich Peter Döhlmann (B, 416), Stephan Kistenmaker (B, 417), Moritz Hagenström (B, 416), Andreas Pringsheim (B, 337), oder Siegmund Köstermann (B, 12) hießen, werden in der Übersetzung mit polnischen VN-Varianten bedacht: Piotr Döhlmann (B, Bd. 2, 74), Stefan Kistenmaker (B, Bd. 2, 75), Maurycy Hagenström (B, Bd. 2, 74), Andrzej ${ }^{10}$ Pringsheim (B, Bd. 2, 6) oder sogar Zygmuś Köstermann $(B, 11)$. Alle genannten Figuren, wobei wir im Roman mit einer großen Menge ähnlicher Fälle zu tun haben, tragen polnische VN-Dubletten, obwohl eine Beibehaltung der Originalformen durchaus denkbar wäre. Dies wäre auch ratsam, da der eingebürgerte Vorname in einem auffälligen Widerspruch zum transkribierten deutschen Familiennamen steht. Der Personenname der Figur verliert dadurch an Einheitlichkeit, was öfters auch weitere inhaltliche und stilistische Verluste mit sich bringt. Folgen der einbürgernden Wiedergabe werden anhand folgender Belege besprochen.

\section{Sigismund Gosch - Zygmunt Gosch}

Sigismund Gosch hat natürlich seine Schwächen... (B, 498)

Zygmunt Gosch ma oczywisicie swoje stabostki... (B, Bd. 2, 143)

${ }^{10}$ Hier ist eine gewisse übersetzerische Inkonsequenz zu bemerken, da derselbe Vorname in bezug auf eine andere Figur in die Zielsprache unverändert übernommen wird: Andreas Giesecke $(B, 54)$ kommt auch in der Übersetzung als Andreas Giesecke $(B, 48)$ vor. 
Die Figur des Maklers Gosch enthält viele Widersprüche und sie schafft zusammen mit anderen bildhaften Randfiguren einen interessanten Hintergrund für die handelnden Hauptgestalten. Die Zerrissenheit der Figur wird in der Uneinheitlichkeit des antithetischen Namens deutlich ${ }^{11}$, der aus einem mehrsilbigen Vornamen und einem einsilbigen Nachnamen besteht. Dabei ist die klangsymbolische Kürze des Familienamens in der AS durch die Länge des VN gesteigert, wodurch der $\mathrm{FN}^{12}$ "fast wie ein Schimpfwort klingt" (Tyroff, 1975:126).

Sigismund ist ein alter deutscher Vorname, eine Nebenform von Siegmund, der auf die Verehrung des heiligen Sigismund, Königs von Burgund (5./6. Jh.), zurückgeht (vgl. Drosdowski 1974:188). Der Vorname evoziert einen höheren gesellschaftlichen Status, da er der älteren Sprachschicht angehört und als Königsname bekannt ist (z.B. Kaiser Sigismund, König von Böhmen 14./15. Jh.). Sigismund wird im ZS-Text durch die polnische Dublette Zygmunt substituiert. Dies bringt Verluste an Fremdheitsatmosphäre mit sich, die in diesem Fall die zielseitige Rezeption beträchtlich beeinflussen. Der Vorname Zygmunt wirkt sehr ,polnisch“, denn man assoziiert ihn mit den polnischen Königen (Zygmunt Stary, Zygmunt August usw.). Demzufolge hängt sein Assoziationsfeld mit der polnischen Geschichte eng zusammen. So verursacht die Substitution eine Änderung des Namenfeldes und darüber hinaus Verluste an AS-Konnotationen: Der VN wird um eine Silbe kürzer, was die Uneinheitlichkeit der Namenstruktur mildert (s.o.).

Auch der Hinweis auf die Seltenheit der alten Namensform ist nicht mehr vorhanden. Obwohl der ZS-Leser die Assoziationen von Sigismund (ältere Sprachschicht, höhere soziale Stellung) nicht erfassen kann, würde der transkribierte Vorname einen Hinweis nicht nur auf die AS-Welt, sondern auch auf die Ungewöhnlichkeit des VN (Kompliziertheit, Mehrsilbigkeit) beinhalten, was für das Verstehen der Figur sehr wichtig ist. Infolge der Substitution werden alle Aspekte der Namenwirkung nivelliert, und es entstehen zielseitige Assoziationen, die der AS-Text nicht enthält.

\section{Bendix Grünlich - Benedykt Grünlich}

Ich zum Beispiel, wenn ich mir diese Bemerkung gestatten darf, (...) heiße wie die

${ }^{11}$ Der antithetische Name signalisiert einen Gegensatz in der Figur signalisiert (vgl. Lamping 1983:47-54).

${ }^{12}$ Der FN ist darüber hinaus redend, da er der mundartlichen saloppen Bezeichnung Gosche, Gosch oder Goschen für 'Mund' gleicht (z.B. „eine große Gosche haben", Duden-Universal, 1989:623). Zuerst macht sich hier das mundartliche Element bemerkbar, das dem Nachnamen einen niedrigen sozialen Status verleiht, was wieder auf die Uneinheitlichkeit der Figur hinweist (im Vergleich zum seltenen VN). Die appellativische Bedeutung bezieht sich auf den Beruf von Gosch, der als Makler wohl viel reden muß (und auch sehr beredt ist). Der transkribierte Name entbehrt aller Bedeutungen und läßt den Träger nur national einordnen. 
meisten meiner Vorfahren Bendix, - ein Name, der ja nur als eine mundartliche Zusammenziehung von Benedikt zu betrachten ist. (B, 80).

- Ja, na przyklad, jezeli wolno mi tu wspomnieć o sobie, mam jak większość moich przodków na imię Benedykt. $(\mathrm{B}, 70)$

Der Vorname Bendix sorgt im Original dafür, daß die Figur des affektiert wirkenden Herrn Grünlich noch vollständiger charakterisiert wird, weil der Vorname einen ziemlich ungewöhnlichen und genauso gekünstelten Eindruck wie sein Träger macht. Außerdem wird im Text die mundartliche Herkunft des seltenen Vornamens von seinem Träger selbst erläutert. Da der Vorname im ZSText in seiner Standardform vorkommt (Benedykt) und man nichts von Bendix hört, läßt der Übersetzer den Kommentar der Figur aus, obwohl er gerade im Fall der Transkription die Befremdlichkeit der Form vollständig erläutern könnte (Bendix sei „eine mundartliche Zusammenziehung von Benedikt"). Dabei ist es kein übersetzerischer Zusatz, sondern eine im Text selbst enthaltene Erläuterung, die den mundartlichen Vornamen auf eine beiderseits bekannte Namensform: Benedikt/Benedykt bezieht.

Der Name Benedikt (zu lat. benedictus - 'gepriesen') ist für die Charakterisierung der Figur des Erbschleichers von Belang: Der Gesamtname Bendix (=Benedict) Grünlich zerfällt somit in zwei Teile, der Vorname ist religiös bestimmt (erinnert auch an den hl. Benedikt von Nursia), und der redende Nachname verrät ein unbestimmtes Wesen (redender Inhalt vom Adjektiv grünlich), was bereits auf die Zweideutigkeit des Charakters hinweist (vgl. auch Tyroff 1975:127f). Außerdem ist Benedikt ein passender Vorname für eine Person, die seine Religiosität vortäuscht und sich als „ein so christlicher junger Mann” (B, 82) bei der Familie Buddenbrook einschmeichelt.

Die im AS-Text enthaltene Erläuterung (Bendix als eine Form von Benedikt) vermag auch diese Assoziationen zu bewahren, ohne daß es notwendig wäre, die Form Bendix direkt mit dem zielseitigen Benedykt zu ersetzen. Dabei könnte die transkribierte Form auch das spezifisch lokale Element bewahren, das dank der Erklärung auch für die ZS-Leser zugänglich wäre. Nun könnte die Bewahrung des fremd klingenden Namens Bendix auch in der Übersetzung die charakterisierende Funktion erfüllen. Die Ersetzung mit Benedykt läßt aber diesen Aspekt aus, denn die auffällige Andersartigkeit der Form Bendix wird völlig nivelliert. Die Substitution ist infolgedessen als Reduktion zu begreifen, da ein Teil des AS-Textes unübersetzt bleibt, wodurch nahezu alle Konnotationen des Vornamens ausbleiben müssen. Das Argument der Anpassung an das zielseitige Deklinationssystem gilt in diesem Fall nicht, da sich der Vorname mühelos im Polnischen flektieren ließe. Es ist verwunderlich, daß die Übersetzerin eine Textstelle willkürlich ausläßt, die ihr bei der Bewahrung der viel- 
schichtigen Form Bendix durchaus behilflich sein könnte. Es ist m.E. kein Zeichen der übersetzerischen Unterlassung, eher ein Zeugnis dessen, daß man als Übersetzer keinen großen Wert auf die Rolle literarischer Eigennamen im Werk legt und daher die im Text enthaltene Hilfe ignoriert.

\subsection{Transkription der Vornamen in den Buddenbrooks}

In der Übersetzung des Romans kann man eigentlich nur vereinzelte Fälle der Vornamen belegen, die deswegen transkribiert werden, weil sie über keine zielseitige Dublette verfügen. Dabei sind es fast ausnahmslos maskuline Vornamen: Gotthold $(B, 13)$, Justus ${ }^{13}(B, 12)$, Jürgen $(B, 184)$, Diederich $(B, 90)$. Die genannten Vornamen können dank dem Konsonantenauslaut in der Zielsprache mühelos flektiert werden. Dies beweist die Tatsache, daß es eigentlich nicht undenkbar wäre, die meisten maskulinen Vornamen zu transkribieren, da sie im Original auf einen Konsonanten ausgehen und somit die zielseitige Deklination ermöglichen. Bei einer konsequenten Transkription der Vornamen wäre es empfehlenswert, die Aussprache deutscher Vornamen in einem Anhang zu erläutern. Dies würde das Lesen erleichtern, da man nicht mehr über unaussprechbare Formen stolpern müßte. Darüber hinaus würde es einen Einblick in die fremde Sprache gewähren und somit einen Erkenntniswert besitzen.

Feminine Vornamen stellen ein viel komplizierteres Problem dar, weil die polnischen femininen Vornamen immer auf ein -a ausgehen. Falls der feminine ASVorname auf einen anderen Vokal oder einen Konsonanten ausgeht, muß seine transkribierte Form im Polnischen unflektiert bleiben; dies geschieht z.B. im Fall Kathi (B), Elsbeth (F) oder Ellen (Z). Bei einer solchen Wiedergabe weist man oft auf die Gefahr hin, daß diese Formen nicht mehr als feminin empfunden werden. Dabei liegen gerade im Deutschen nicht selten Vornamen vor, die eindeutig auf eine zielseitige feminine Namendublette hinweisen (Karoline, Hermine, Friederike, Sophie, Henriette etc.). Darüber hinaus darf man die Rolle des Kontexts (und den Leser selbst) nicht unterschätzen, denn falsche Assoziationen können in Zweifelsfällen durch den Zusammenhang korrigiert werden (vgl. Lietz 1992:180). Zwar sind solche Vornamen von der ZS-Flexion begrenzt, aber warum sollte man auch hier die Transkription nicht wagen? Es existieren in der Übersetzung Fälle (s.o.), wo

${ }^{13}$ Der pietistische Vomame Justus wird auch nicht konsequent wiedergegeben. An manchen Stellen bleibt der Vorname unverändert: „Nun wendet sich der Pastor an die Paten (...). Justus Krōger ist der eine...” (B, 338). / „Teraz pastor zwraca się do ojców chrzestnych (...) Jednym z ojców jest Justus Krōger..." (B, Bd. 2, 7). Aber er kommt auch als Justyn vor: ,'Guten Abend, Justus', sagte die Konsulin. ,Geht es dir gut?”" (B, 211) / „Dobry wieczór, Justyni - powiedziała konsulowa - Co u ciebie stychac'?" (B, 182). Wohlgemerkt ist Justus, der einzige pietistische Vorname, dessen semantischer Gehalt dank seiner lateinischen Wurzeln auch in der Zielsprache semantisch durchsichtig wäre (im Gegensatz zu Leberecht oder Gotthold). 
dies durchgeführt wird und sich als eine gelungene Lösung bewährt; z.B. über den VN Elsbeth schreibt die Übersetzerin des Doktor Foustus ${ }^{14}$, daß der Vorname keineswegs als Elżbieta polonisiert werden darf (vgl. Kurecka 1970:88).

Warum sollte man also auch andere feminine und maskuline Vornamen nicht als Zeichen auffassen, deren Fremdheit in der Übersetzung bewahrt werden soll? Dies könnte um so mehr aus dem Grnnd geschehen, weil vieles von der Fremdheit und Andersartigkeit der AS-Welt im Übersetzungsprozeß zwangsläufig verlorengeht. Man kann sich dabei zusätzlich fragen, was weniger störend wirkt: Vornamen, die sich nicht flektieren lassen, oder Vornamen, von durchaus „fremden”, „hanseatischen" Figuren getragen, die jedoch wie einheimische, vertraute Vornamen klingen: Katarzyna, Zofia, Henryka? Heißt das nicht, daß das Werk "seiner kulturellen Identität beraubt" wurde (vgl. Lietz 1992:180)?

Die beibehaltenen deutschen Vornamen können nämlich auch in der Zielsprache Zeichen der fremden Welt sein und das Spezifische dieser Welt betonen. Die Transkription der Vornamen, könnte im Roman ein wirksames stilistisches Mittel sein, das Assoziationen des Fremden in der Übersetzung evozieren und auch die mehrfachen Verluste auf anderen Ebenen (z.B. die völlige Nivellierung des Plattdeutschen in B) kompensieren könnte.

Im Original erkennt er [der Leser] die kulturellen Elemente als Identitätssignale seiner eigenen Welt und der Welt der Literatur; in der Übersetzung muß der potentielle Leser diese Elemente als Signal der Kulturfremdheit entschlüsseln.

(Legeżnnska 1983:88; Üb. E.P.)

Das Postulat der Transkription muß aber noch angesichts der Problematik der Wiedergabe von Hypokoristika behandelt werden.

\subsection{Wiedergabe der Hypokoristika}

Polnische Übersetzer stehen oft vor dem Problem, wie sie deutsche Koseformen wiedergeben, damit 1. ihre stilistische Funktion bewahrt bleibt (das Diminitivum soll als solches von den Lesern erkannt werden) und 2. eine in der Zielsprache erkennbare Koseform nicht zu zielsprachlich dünkt. Die Analyse der drei Romane (B, Z, F) von Thomas Mann zeigte, daß sich die Übersetzer in den meisten Fällen für die Wiedergabe der Koseform in der Zielsprache mit Hilfe der ZS-Sprachmittel entscheiden. Die Ausdruckskraft der Hypokoristika bleibt

${ }^{14}$ In Doktor Faustus kommen zahlreiche feminine Vornamen vor, die nicht auf ein - $a$ ausgehen: Waltpurgis, Hanne, Bärbel, obgleich viele andere adaptiert werden: Ursel zu Urszula, Helene zu Helena, Clementine zu Klementyna. 
dann zwar bewahrt, aber zwangsläufig haben wir in der Übersetzung mit substituierten Vornamenformen zu tun. Dies mögen folgende Textbelege bezeugen, in denen deutsche Hypokoristika mit der Endung -chen gebildet werden:

Wer von den jungen Damen hat noch nicht gebadet? Mariechen, Julchen, Luischen? (B, 111)

Która z panien jeszcze się nie kapala? Marysia, Julcia, Luizka? (B, 97)

Klothildchen hat tücht' $\mathrm{g}$ geholfen in der Küche. $(\mathrm{B}, 10)$

Tyldzia chwacko pomagala w kuchni. (B, 9)

Es kam aber hinzu, daß er an die Person des Rechtsanwalts nicht denken konnte, ohne auch Fränzchen Oberdank mit einzubeziehen... (Z, 423)

Ponadto nie mógl myśleć o osobie adwokata nie wspominajac przy tym Franeczki Oberdank...? $(\mathrm{Z}, 516)$

Die Koseformen haben in den obigen Belegen unterschiedliche Funktionen. Im Fall von Klothildchen bezieht sich die Koseform auf ein kleines Kind; die Anrede „Mariechen, Julchen, Luischen” steht für junge Mädchen aus der vornehmen Travemünder Gesellschaft, die auf eine väterliche Weise zum Baden aufgefordert werden; im Fall von Fränzchen ist die Form eher ironisch gebraucht, da es sich um eine Liebesaffäre zwischen einem älteren Rechtsanwalt und einem jungen Mädchen handelt. Um ihre Funktion zu bewahren, sollen die Diminutiva mit entsprechenden zielseitigen Sprachmitteln gebildet werden: Deutsche Diminutiva, die auf -chen ausgehen, werden also durch polnische Koseformen der Namendubletten ersetzt. So wird Klothildchen zu Tyldzia, Mariechen zu Marysia und Fränzchen zu Franeczka.

Dabei darf jedoch die Wiedergabe der Hypokoristika nicht ohne Bezug auf andere Vornamenformen und die gesamte Fremdheitsatmosphäre betrachtet werden. Auch hier ist die Stellungnahme zur Frage: "Substituieren/adaptieren oder transkribieren?" von Bedeutung. Wenn in einer Übersetzung Substitution und Adaptation von Vornamen vorherrschen, so ist auch die Entscheidung, Hypokoristika zu adaptieren/substituieren, nicht überraschend. Wenn man jedoch das transkribierende Verfahren in Erwägung ziehen würde, dann würden sich weitere Fragen häufen: Soll man den Namen samt der Endung -chen transkribieren, die zielseitig nichts bedeutet, aber sich vielleicht in einer kurzen Anmerkung erläutern ließe oder wenigstens aus dem Kontext erschließbar wäre (der Bezug auf die Vollform ist dabei durchaus deutlich)? Oder soll man die Koseformen ohne Kommentare transkribieren und Verluste bei der Namenstilistik in Kauf nehmen?

Die Beibehaltung der deutschen Endung in der Koseform kann man lediglich in Doktor Faustus vorfinden, wo auch in der Zielsprache von Aenchen und Riekchen die Rede ist: 
Zwei Jahre spāter folgte ihr ein Zwilingspärchen, Mädchen wiederum, die (...) auf den Namen Annchen und Riekchen getauft wurden. (F, 329)

Dwa lata później pojawily się jeszcze bliżnięta, znowu dziewczynki, które (...) zostaly ochrzczone imionami Aennchen i Riekchen. (F, 302)

Da dies jedoch der einzige Fall ist, in dem man die deutsche Diminutivendung auch in der Zielsprache findet, kann man ihn eher als eine die Regel bestätigende Ausnahme verstehen. Dabei ist dieser Beleg nicht repräsentativ, denn man kann hier die Funktion der Hypokoristika nicht erkennen: Es gibt im Text keine Vollformen zu diesen Vornamen, die ihr Bezugspunkt wären und das Verkleinerte erkennen ließen.

Dabei gibt es im deutschen Text Kurzformen der Vornamen, die als Koseformen fungieren. Ihre Transkription (Adri zu Adrian, Rudi zu Rudolf, in F) verursacht keine Schwierigkeiten: Sie werden in ihrem Bezug auf die jeweiligen Vollformen (Bewahrung der Referenz) und in ihrer Diminutivfunktion (Bewahrung der Namenstilistik) erkannt. Vielleicht kann nur das inkonsequente Verfahren in bezug auf den Vormamen Nikolaus die Verwirklichung der Referenzfunktion gefährden, denn die Vollform wird adaptiert (Mikołaj), wobei sich die transkribierte Kurzform Niko (F, 51) auf die im ZS-Text abwesende Form Nikolaus bezieht. Auch die femininen Kurzformen können transkribiert werden, z.B. Elly (Z), die in beiden Sprachen diese Form bewahrt und demzufolge im Polnischen nicht deklinierbar ist. Die Kurzform Tony (B) zur Vollform Antonie ${ }^{15}$ wird demgegenüber adaptiert (Tonia), wahrscheinlich um die Deklination zu ermöglichen. Bei der Entscheidung über das anzuwendende Verfahren der Wiedergabe von Hypokoristika sollten auch Fälle bedacht werden, in denen die Bewahrung des emotionalen Wertes der Koseform das Postulat zur Bewahrung der Fremdheit überwiegt.

Sesemi: Im folgenden Beispiel haben wir es mit der Lallform Sesemi zu tun, die sich auf Therese Weichbrodt, eine ältliche Leiterin der Mädchenpension, bezieht:

Als Kind (...) hatte Therese Weichbrodt sich selber ,Sesemi' genannt. (B, 70)

Jako dziecko (...) nazwala się sama Teteniq. (B, 61)

Die Bewahrung der Form Sesemi könnte nicht den komischen Effekt wiedergeben, der aus der Verbindung der Lallform Sesemi (Sprachregister: Kindersprache) mit der ernsten Funktion der Leiterin der Mädchenpension entsteht ${ }^{16}$. Die

${ }^{15}$ Wohlgemerkt wird die deutsche Vollform im Polnischen nicht mit der üblichen Dublette Antonina, sondern mit Antonia ersetzt: „Dzis jako forma žeńska imienia Antoni występuje raczej Antonina, a Antonia poszła w zapomnienie" (Fros/Sowa 1997:227).

${ }^{16}$ Die Leiterin trägt dabei noch den bildhaften, redenden Nachnamen Weichbrodt, der den ironisch-komischen Effekt steigert und der zielseitig schweigen muß. 
Übersetzerin sucht nach einer entsprechenden Lallform zum Vollvornamen Therese - Teresa und wählt die zielseitig bekannte Form Tetenia, die sich bei der Bewahrung der Namenstilistik als sehr gelungen erweist.

Tonnchen (Erikachen, Hannochen), mein Kindchen: Einen besonders komplizierten Fall stellen Hypokoristika dar, die zusammen mit den appellativischen Diminutiven vorkommen:

Tonnchen, mein Kindchen, braucht keine Sorgen haben. $(\mathrm{B}, 84)$

Toniuchno, córuchno, nic się nie martw. (B, 83)

Das gereimte Syntagma „Tonnchen, mein Kindchen” bewirkt, daß die Koseform beider Elemente: Proprium und Appellativum mit den zielseitigen Sprachmitteln gebildet und auch gereimt wiedergegeben wird: Toniuchno, córuchno $^{17}$. Dies bringt die Vertrautheit und Zuneigung der redenden Person zu Tony Buddenbrook auf eine sehr gelungene Weise zum Ausdruck. Die Form Toniuchno macht jedoch einen stark zielseitigen Eindruck und beeinflußt auch Übersetzungen weiterer Syntagmen, die gleiche Struktur haben.

... so sagte Mamsell Jungmann: Erikachen, hier zieht's. $(\mathrm{B}, 208)$

... panna Jungmann mówila: - Eruchna, tu wieje! (B, 179)

Die Notwendigkeit der Bewahrung der Koseform Erikachen in der ZS mit Hilfe entsprechender zielseitiger Sprachmittel bezieht sich auf die vorhergehende Entscheidung, Tonnchen als Toniuchna wiederzugeben. Nun kann die Substitution der Form Erikachen nicht mit jeder Verkleinerungsform der adaptierten Vollform Eryka (z.B. Eryczka) ersetzt werden.

Zum wiederholten Mal erscheint Ida Jungmann mit ihrer charakteristischen Sprechweise auf der Bildfläche, wenn sie den kleinen Hanno betreut, dessen Vorname wiederum ,nach allen Regeln der Kunst” mit der Diminutivendung -chen zu Hannochen verkleinert wird.

Dann aber sagte Ida Jungmann: Komm Hannochen; müssen gehen... (B, 539)

Nächst's Jahr wieder, Hannochen (B, 541)

Ale potem Ida Jungmann mówila: - Chodź, Januchna, trzeba już išć... (B, Bd. 2, 177)

${ }^{17}$ Kindchen wird also als córuchno wiedergegeben, aber wie soll man mit dem Vornamen umgehen? Hier macht sich der Unterschied zwischen Propria und Appellativa bemerkbar: PN sind Zeichen der fremden Welt und man darf mit ihnen nicht wie mit Appellativa verfahren, weil sie dann in ihrem Ausdruck verflachen und weil ihre Funktion mißdeutet werden kann. Es ist also nicht nur die Frage, wie man die Koseform wiedergeben soll, sondern auch die der Grenze zwischen Eigennamen und Gattungsnamen. 
- Na przyszły rok znowu, Januchna. (B, Bd. 2, 179)

Sei still, Hannochen, mein Jungchen, brauchst nichts hersagen... (B, 412)

Cicho, Januchna, synuchna, nie potrzebujesz nic deklamować... (B, Bd. 2, 70)

Für die polnische Übersetzerin wird jedoch Idas Verkleinerung zu einer beträchtlichen Schwierigkeit, die sich in den zwei unterschiedlichen Verfahren bei der Übersetzung der Namensformen niederschlägt: Adaptation der Vollform (aus Johann ist Jan geworden) und Transkription der Kurzform (Hanno). Die AS-Koseform Hanno bezieht sich auf die AS-Vollform Johann, die in der Zielsprache mit Jan adaptiert wird, wodurch der direkte und sichtbare Bezug des Hypokoristikums auf die Grundform in der Übersetzung nivelliert wird. Die transkribierte Form Hanno ließe sich aber nach dem Muster nur schwer verkleinern und könnte wohl zu Mißverständnissen führen, da sie z.B. als Hanuchna einem femininen Vornamen gleich wäre. Die Übersetzerin greift auf die Vollform zurück (im Gegensatz zu Ida, die ihre Verkleinerung von der Kurzform ableitet), die in der Übersetzung adaptiert wird. So haben wir neben der transkribierten Kurzform Hanno eine Verkleinerung der adaptierten Form: Januchna (wie Toniuchna, Eruchna). Zwar wird hier der expressive Wert der Koseform bewahrt und Idas Sprechweise auch an dieser Stelle wiedergegeben, aber es kommt hinzu, was dank der Anwendung der Transkription (Hanno) vermieden wurde: Die Form Januchna mutet äußerst polnisch an und ruft unerwartete Konnotationen hervor. Es entstehen Verschiebungen in der nationalen und auch sozialen Zuordnung, da die Koseform eher an einen polnischen Dorfjungen als an einen Konsulsohn erinnert.

Die drei Koseformen Tonnchen, Hannochen, Erikachen befinden sich in einem deutlichen Verhältnis zueinander, so daß ihre Ähnlichkeit auch zielseitig konsequent beibehalten werden muß. Die eingebürgerte Koseform muß mit den anderen Formen kohärent bleiben, was jedoch zu einer Form führt, die stark zielseitig dünkt (in bezug auf Appellativa: mamuchna, córuchna usw.). Andererseits wird jedoch die charakteristische Sprechweise Ida Jungmanns ${ }^{18}$ bewahrt, die aus Westpreußen stammte ${ }^{19}$ und dazu ab und zu auch einige polnische Wörter gebrauchte ${ }^{20}$. So können in diesem Fall die Formen auf die Spezifik der Figur zurückgeführt werden und zur Charakteristik der Figur beitragen, auch wenn man dies als einen Fall der overtranslating verstehen kann.

${ }^{18}$ Hier ist auch die Tatsache wichtig, daß sich Jungmanns Redeweise durch den ganzen Roman zieht, deswegen muß der ersten Übersetzung konsequent eine ähnliche folgen.

19 „...sagte Mamsell Jungmann und schnurrte das $r$ in der Kehle, denn sie hatte es ursprünglich überhaupt nicht aussprechen können." (B, 10).

${ }_{20}, \ldots .$. worauf Ida vor freudigem Schreck etwas Polnisches antwortete, das klang wie: ,Meiboschekochhanne!'” (B, 274). 


\section{Ist ein einheitliches Verfahren in bezug auf die Wiedergabe der Vornamen möglich?}

Die Analyse der Vornamen im Roman Buddenbrooks hat gezeigt, daß Vornamen vorwiegend adaptiert werden und daß Transkription eher selten eingesetzt wird. In der theoretischen Einführung wurde im Gegensatz dazu festgestellt, daß Vornamen ein integraler Teil der AS-Welt sind und nur als transkribierte Formen in der ZS-Empfindung dieser Welt weiterhin angehören können (vgl. 2.1). Die Befürwortung der Transkription betont nämlich die Möglichkeit, dank den transkribierten Vornamen einen Einblick in die fremde Welt, ihre Kulturspezifik und Atmosphäre zu gewähren. Transkribierte Vornamen tragen dazu bei, die Fremdheitsatmosphäre auch in der Übersetzung beizubehalten und die durch die Namengebung vermittelte Kultur und Tradition zu bewahren. In diesem Sinne spricht Nowakowska-Kempna von der Pflicht der Transkription (vgl. 1978:112; s. Fußnote 7).

Vornamen sollen also wie Familiennamen betrachtet werden, die laut heutiger Übersetzungsnorm nicht substituiert werden. Das, was heutzutage in bezug auf FN eine allgemein akzeptierte Regel der Wiedergabe ist (man ersetzt also den Namen Hans Schmitt nicht mit der scheinbar adäquaten Form Jan Kowalski), ist immer noch eine Ausnahme im Bereich der VNWiedergabe.

Natürlich kann man dabei nicht übersehen, daß Adaptation ihre Gründe hat. Die wichtigste Voraussetzung der Adaptation ist die Notwendigkeit der VN-Flexion im Polnischen. Die meisten Übersetzer möchten nicht gegen die Sprachregeln verstoßen und entscheiden sich für die Einbürgerung. Dieses Verfahren wird jedoch ziemlich inkonsequent eingesetzt, da sich in den besprochenen Übersetzungen der Romane von Thomas Mann auch Vornamen finden lassen, die in der Zielsprache nicht flektiert werden können. Sie sind ein guter Beweis dafür, daß es denkbar ist, einen transkribierten Vornamen an die zielseitige Sprachstruktur nicht anzupassen ${ }^{21}$.

Eine andere Beschränkung des Postulats der Transkription stellen Hypokoristika dar, die über eine gewisse Namenstilistik verfügen. Ihr emotionaler Wert kann mit zielseitigen Sprachmitteln bewahrt werden, da ASHypokoristika als solche in der Zielsprache nicht immer erkannt werden. Dies betrifft in erster Linie Diminutiva, die mit den Endungen -chen und -lein gebildet werden. Die Funktion anderer Diminutiva, die den Kurzformen gleichen

${ }^{21}$ Dies kann man z. B. in modernen Übersetzungen aus dem Englischen feststellen, wo man eine Adaptation George mit Jerzy nicht mehr wagen würde. 
(Niko zu Nikolaus, Adri zu Adrian, Rudi zu Rudolf, F), ist in der Zielsprache mühelos erkennbar. Anhand der Textbelege läßt sich feststellen, daß Hypokoristika ein Faktor sind, der die Forderung nach der Anwendung der Transkription beträchtlich relativiert, obwohl man gleichzeitig bei einer konsequenten Transkription annehmen könnte, daß auch Hypokoristika aus dem Kontext und in ihrem Bezug auf die Vollformen erkennbar wären (s.o.).

Die praktisch eingesetzte Transkription der Vornamen zeigt ${ }^{22}$, daß transkribierte Vornamen die AS-Welt erfolgreich evozieren können: Ihre Verwendung betont die Fremdheit und hebt das Lokalkolorit hervor. Dabei wird dieser Gewinn durch Deklinationsschwierigkeiten kaum in Frage gestellt. Die übersetzerische Praxis beweist, daß eine Transkription der Vornamen trotz obiger Vorbehalte durchaus möglich und nützlich ist.

Die Analyse des Namenkorpus zeigt gleichzeitig, daß es Fälle gibt, in denen Adaptation notwendig ist, da sie gerade die Bewahrung der Bedeutsamkeit garantiert. So werden hebräische Vornamen Elia und Rahel $(Z)$ im Polnischen adaptiert (Eliasz und Rachela), damit sie eindeutig auf die jüdische Herkunft Leo Naphtas hinweisen können. Ein anderer Fall ist die Adaptation des Namens Anton (als Antoni), die das sozial zuordnende Element in der Übersetzung bewahrt (B), und des Namens Alois Permaneder (B), der als Alojzy Permaneder wenigstens die konfessionelle (katholische), wenn nicht die lokale (bairische) Zuordnung wiederzugeben vermag. Man kann jedoch sagen, daß solche Einzelfälle das Postulat der Transkription von AS-Vornamen im allgemeinen nicht aufheben.

Es ist eher ein Beweis, daß man sich im Bereich der Wiedergabe literarischer Personennamen vor allzu großen Verallgemeinerungen hüten soll, denn es gibt nicht „,die” Methode der VN-Wiedergabe, die in allen Fällen anzuwenden wäre. Vielmehr muß man alle Funktionen des Vornamens in Betracht ziehen und das gegebene Verfahren auch in bezug auf die Ganzheit des Textes prüfen. Somit läßt sich zusammenfassend feststellen, daß die Wiedergabe eines literarischen Namens immer als ein konkreter Fall überlegt werden muß. Es lassen sich keine allgemeinen Regeln aufstellen, die für alle Namen und alle Funktionen literarischer Namen gültig wären. Man darf aber postulieren, die Methode der Transkription zu bevorzugen, weil sie wichtige Funktionen der Vornamen zu bewahren vermag und auch einen erkenntnisreichen Einblick in die fremde Kultur ermöglicht. Gleichzeitig jedoch muß man die Grenzen des Verfahrens sehen, die in der Struktur der Sprache und in den Funktionen des jeweiligen Vornamens zu suchen sind.

22 Dies kann man v.a. am Beispiel des Romans Doktor Faustus beobachten, der relativ viele transkribierte Vornamen (auch feminine) enthallt: Bärbel, Hanne, Marie, Georg, Elsbeth, Jonathan. 


\title{
QUELLENVERZEICHNIS
}

\author{
WERKE VON THOMAS MANN \\ (MIT DEN VERWENDETEN ABKÜRZUNGEN)
}

(B) Buddenbrooks. Frankfurt a.M.: Fischer Verlag, 1987.

Buddenbrookowie. Üb. E. Librowiczowa. Warszawa: Ksiązka i Wiedza, 1988.

(F) Doktor Faustus. Frankfurt a. M.: Fischer Verlag, 1975.

Doktor Faustus. Üb. M. Kurecka, W. Wirpsza. Warszawa: Czytelnik, 1985.

(Z) Der Zauberberg. Berlin/Weimar: Aufbau-Verlag, 1974.

Czarodziejska góra. Üb. J. Kramsztyk (Bd. 1), J. Łukowski (Bd. 2). Warszawa: Warszawski Dom Wydawniczy, 1992.

\section{SEKUNDÄRLITERATUR}

Bubak, Józef (1998): Zmiany w zasobie imion chrzestnych współczesnych Polaków. In: JakusBorkowa, Ewa/Nowik, Krystyna (Hg.). Najnowsze przemiany nazewnicze. Warszawa: Energeia (47-57).

Cieślikowa, Aleksandra (1996): Jak ,ocalic w tłumaczeniu' nazwy własne? In: Filipowicz-Rudek, Maria; Konieczna-Twardzikowa, Jadwiga (Hg.). Między oryginatem a przekładem. Przekład, jego tworzenie się $i$ wptyw. Bd. 2. Kraków: Universitas (311-323).

Debus, Friedhelm (1997): Eigennamen in der literarischen Übersetzung. In: Grammatica Ianua Artium. Festschrift für Rolf Bergmann zum 60. Geburtstag. Glaser, Elvira/Schlaefer, Michael (Hg.). Heidelberg: Universitätsverlag C. Winter (393-405).

Grodzinsski, Eugeniusz (1973): Zarys ogólnej teorii imion własnych. Warszawa: PIW.

Güttinger, Fritz (1963): Zielsprache. Theorie und Technik des Übersetzens. Zürich: Manesse Verlag,

Jakus-Borkowa, Ewa (1986): Nazwy własne we wspótczesnym języku polskim. Leipzig: KarlMarx-Universităt.

Kalverkämper, Hartwig (1978): Textlinguistik der Eigennamen. Stuttgart.

Krüger, Dietlind (1997): Wort und Name im deutsch-slavischen Sprachkontakt. In: Hengst, Karlheinz; Krüger, Dietlind, ... (Hg.). Ernst Eichler von seinen Schülern und Freunden (Sonderdruck). Köln/Weimar/Wien: Böhlau Verlag (464-480).

Krusche, Dietrich (1985): Literatur und Fremde. Zur Hermeneutik kulturräumlicher Distanz. München: Idicum-Verlag (Zit. in Markstein, 1995).

Kurecka, Maria (1970): Diabelne tarapaty. Poznan: Wydawnictwo Poznanskie.

Lamping, Dieter (1983): Der Name in der Erzählung. Zur Poetik des Personennamens. Bonn: Bouvier Verlag (Wuppertaler Schriftenreihe Literatur, Band 21).

Legezyñska, Anna (1983): Thumacz i jego kompetencje autorskie. In: Martuszewska, Anna; Sławiński, Janusz (Hg.). Autor - Podmiot literacki - Bohater. $Z$ dziejów form artystycznych w literaturze polskiej. Wrocław/Warszawa/...: Ossolineum (Polska Akademia Nauk, Instytut Badan Literackich, Tom LXIII) (77-93).

Lietz, Gero (1992): Eigennamen in der norwegischen Gegenwartssprache. Probleme ihrer Wiedergabe im Deutschen am Beispiel belletristischer Texte. Frankfurt a.M./Bern/...: Peter Lang Verlag (Europāische Hochschulschriften, Reihe XXI Linguistik, Bd. 109).

Markstein, Elisabeth (1995): Das Fremde wird Buch. In: Lebende Sprachen, 3. (97-101). 
Neubert, Albrecht (1973): Name und Übersetzung. In: Der Name in Sprache und Gesellschaft. Beiträge zur Theorie der Onomastik. Berlin: Akademie-Verlag (74-80).

Newmark, Peter (1981): Approaches to Translation. Oxford.

Niesporek, Bernadeta (1986): Ze stylistycznej problematyki nazewnictwa w Trylogii Henryka Sienkiewicza. In: Język artystyczny, Bd. 4. Katowice: Uniwersytet Sląski (97-116) (Prace naukowe Uniwersytetu Sląskiego, nr 739).

Nowakowska-Kempna, Iwona (1978): Pozycja nazw własnych w przekładzie dzieła literackiego. In: Język artystyczny, Bd 1. Katowice: Uniwersytet Slaski (97-116) (Prace naukowe Uniwersytetu Sląskiego, nr 238).

Schleiermacher, Friedrich (1973): Über die verschiedenen Methoden des Übersetzens. In: Störig, Hans Joachim (Hg.). Das Problem des Übersetzens. Darmstadt: Wissenschaftliche Buchgesellschaft (Wege der Forschung, Band VIII) (38-70).

Seibicke, Wilfried (1982): Die Personennamen im Deutschen. Berlin/New York: Walter de Gruyter.

Seutter, Konstanze (1996): Eigennamen und Recht. Tübingen: Niemeyer (Reihe: Germanistische Linguistik, 172).

Shin, Kwang Sook (1980): Schichtenspezifische Faktoren der Vornamengebung. Empirische Untersuchung der 1961 und 1976 in Heidelberg vergebenen Vornamen. Frankfurt a.M./Bern/...: Peter Lang Verlag,. (Europäische Hochschulschriften: Reihe 1, Deutsche Sprache und Literatur).

Thies, Henning (1978): Namen im Kontext von Dramen. Studien zur Funktion von Personennamen im englischen, amerikanischen und deutschen Drama. Frankfurt a. M./Bern/Las Vegas (Sprache und Literatur 13).

Tyroff, Siegmar (1975): Namen bei Thomas Mann in den Erzählungen und den Romanen Buddenbrooks, Königliche Hoheit, Der Zauberberg. Frankfurt a.M./Bern: Peter Lang (Europäische Hochschulschriften I, 102).

Umińska-Tyton, Elżbieta (1985): Formy pochodne imion derywowane sufiksalnie (na przykladzie imion łódzkich). In: Studia językoznawcze 10/1985. Wrocław: Ossolineum (107-155).

Witkowski, Teodolius (1964): Grundbegriffe der Namenkunde. Berlin: Akademie-Verlag.

Wolffsohn, Michael; Brechenmacher, Thomas (1999): Die Deutschen und ihre Vornamen. 200 Jahre Politik und öffentliche Meinung. München/Zürich: Diana Verlag.

Zimmer, Rudolf (1981): Probleme der Übersetzung formbetonter Sprache. Tübingen (Beihefte zur Zeitschrift für romanische Philologie, Bd. 181).

\section{NACHSCHLAGEWERKE}

Drosdowski, Günther (1974): Duden-Lexikon der Vornamen. Mannheim/Wien/Zürich: Dudenverlag. Fros, Henryk; Sowa, Franciszek (1997): Księga imion i śwıętych, Bd. 1-2. Kraków: Wydawnictwo WAM. 